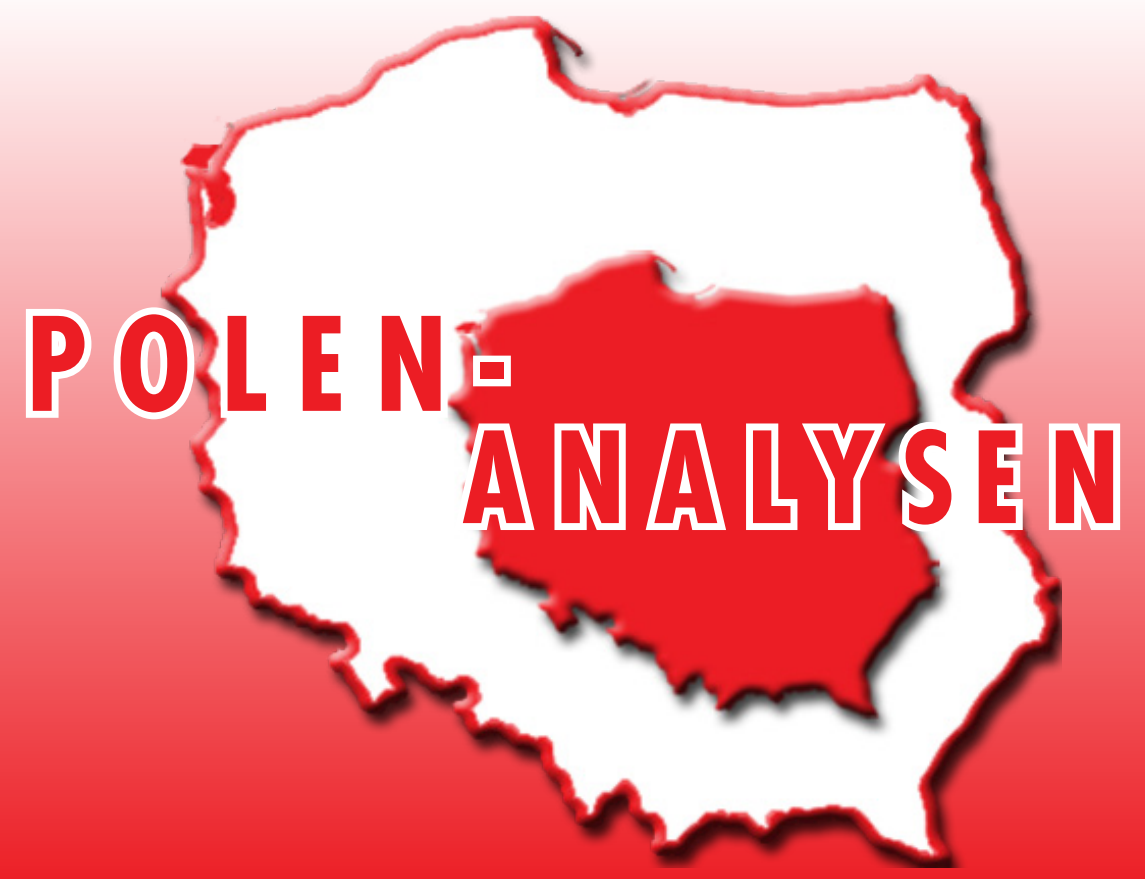

www.laender-analysen.de/polen

\title{
ŐSTLICHE PARTNERSCHAFT
}

- ANALYSE

Östliche Partnerschaft - Perspektiven einer europäischen Ostpolitik

Cornelius Ochmann, Berlin

- TABELLEN UND GRAFIKEN

Der Außenhandel Polens nach Ziel- und Herkunftsländern,

Januar-September 2009

Sympathie- und Antipathiewerte gegenüber ausgewählten Nationen

- DOKUMENTATION

Außenminister Radosław Sikorski im Sejm über die Grundzüge der polnischen Außenpolitik 2010

CHRONIK

Vom 1. bis zum 14. Juni 2010 


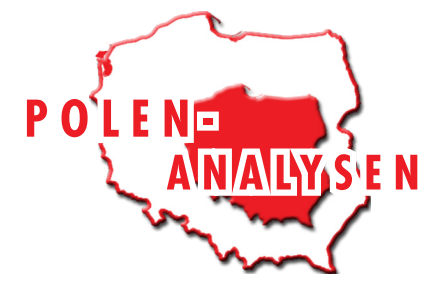

polen-analysen $72 / 10$

Analyse

\section{Östliche Partnerschaft - Perspektiven einer europäischen Ostpolitik}

Cornelius Ochmann, Berlin

\section{Zusammenfassung}

Nach der tragischen Flugzeugkatastrophe in Smolensk am 10. April 2010 ist es zu einem positiven Durchbruch in den polnisch-russischen Beziehungen gekommen. Die Anteilnahme der russischen Bevölkerung und Staatsführung an der Trauer der Polen bestätigte die bereits früher eingesetzte Annäherung der beiden Völker. Die politischen Folgen sind noch nicht absehbar, allerdings lassen sich einige Tendenzen erkennen. Am 24. Mai 2010 fand in Zoppot (Sopot) das informelle Treffen der Außenminister der Östlichen Partnerschaft statt, bei dem nach einjährigem Bestehen eine Bilanz gezogen wurde. Diese ist sehr mager ausgefallen. Außer der Gründung einer "Gruppe der Freunde der Östlichen Partnerschaft« wurden der Öffentlichkeit keine konkreten Fortschritte präsentiert. Am 5. Juni 2010 schlug Bundeskanzlerin Angela Merkel nach zehnstündigen Gesprächen mit dem russischen Präsidenten Dimitri Medwedew die Verstärkung der Kooperation im Bereich der Sicherheit zwischen der Europäischen Union und Russland vor. Auch in diesem Fall sind die Auswirkungen noch unklar. Im Zusammenhang mit der politischen Entwicklung in den Nachbarstaaten der EU, d. h. in der Ukraine und in Belarus, stellt sich die Frage, ob die Östliche Partnerschaft die richtige Strategie für die Gestaltung einer europäischen Ostpolitik ist.

$\mathrm{D}$ Östliche Partnerschaft wurde auf polnischschwedischen Vorschlag als EU-Strategie vom EU-Rat am 28. Mai 2008 verabschiedet und beim Gründungsgipfel am 7. Mai 2009 in Prag bestätigt. Das Ziel der Östlichen Partnerschaft war die konzeptionelle Neugestaltung der Beziehungen der erweiterten EU zu ihren östlichen Nachbarstaaten. Noch während der Ausarbeitung der Strategie brach im August 2008 der Georgien-Krieg aus und veränderte die Rahmenbedingung der gesamten Ostpolitik der EU. Im Januar 2009 stoppte Russland die Gaslieferungen an die Ukraine und fror auf diese Weise die Beziehungen zur EU ein. Entsprechend reagierte die EU mit dem Prager Gipfel zur Östlichen Partnerschaft. Im Verlauf des Jahres änderte sich allerdings die Lage - die Beziehungen zu Russland verbessern sich langsam, aber stetig. Die Östliche Partnerschaft, die finanziell nur mit 600 Millionen Euro ausgestattet ist, soll die reformorientierten Länder im Osten Europas in ihren Bemühungen Richtung Demokratie und Marktwirtschaft unterstützen. Sie sieht sogar langfristig die Möglichkeit der Abschaffung der Visumspflicht vor. Dies ist seit dem Beitritt der mitteleuropäischen Staaten zum Schengenraum im Dezember 2007 eines der symbolträchtigsten Probleme an der Ostgrenze der EU. Schließlich sieht der Vorschlag eine Reihe von Kooperationsfeldern vor, wie Demokratieförderung, Unterstützung der Rechtsstaatlichkeit, Regulierung von Migration, Belebung des Handels und des Tourismus, Verbesserung der Verkehrsanbindungen, Kooperation im Umweltbereich und Studentenaustausch. Hinzu kommen Sondermaßnahmen für
Belarus, die sich unter Umgehung der staatlichen Kontrollorgane direkt an belarussische Staatsbürger richten. Auch Russland wird eingeladen, sich an einzelnen Projekten zu beteiligen. Nach anfänglicher Zurückhaltung bzw. Ablehnung der Östlichen Partnerschaft zeigt sich Russland bereit, an einzelnen Projekten mitzuwirken. Die Östliche Partnerschaft und das Partnerschafts- und Kooperationsabkommen mit Russland sind die Säulen der Neuen Ostpolitik der EU.

\section{Grundlagen der deutschen Politik gegenüber den östlichen Nachbarn der EU}

Die Rede des damaligen Außenministers Frank-Walter Steinmeier am 4. März 2008 formuliert die Grundlage der deutschen Osteuropapolitik. Im Vordergrund steht die Russlandpolitik, die an die Ostpolitik von Willy Brandt anknüpft. Der Architekt der Brandtschen Ostpolitik, Egon Bahr, hatte wiederholt die Verbindung zwischen der deutschen Ostpolitik der 1960er und 70er Jahre und der EU-Ostpolitik betont. In der Grundsatzrede von Steinmeier sollte "Wandel durch Annäherung" nun von der "Annäherung durch Verflechtung" abgelöst werden. Die Mehrheit der politischen Elite in Berlin nahm diese Strategie wohlwollend auf, und bis heute wurde sie von der neuen schwarz-gelben Regierung nicht in Frage gestellt.

Während eines privaten Besuchs beim polnischen Außenminister Radosław Sikorski Anfang April 2008 wurde Steinmeier mit den polnischen Plänen für eine Östliche Partnerschaft konfrontiert. Einen Monat nach seiner programmatischen Rede zur Europäischen Ost- 


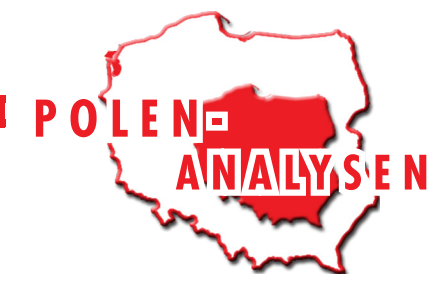

polen-analysen $72 / 10$

politik war er jedoch nicht bereit, den polnischen Vorschlag mitzutragen. Steinmeier stellte die Beziehungen zu Russland in den Vordergrund und sah den polnischen Vorschlag als Gegenvorschlag an: „Wir haben während unserer Präsidentschaft eine EU-Strategie für diese Region entwickelt, die ein breites Spektrum der Zusammenarbeit eröffnet: Energiepartnerschaft, Ausbau der Handelsbeziehungen, gemeinsamer Kampf gegen Drogen und Terrorismus, Förderung rechtsstaatlicher Strukturen, Bildung und Ausbildung, effektive Nutzung der Wasserressourcen «, so Steinmeier in seiner programmatischen Rede und: „Eine moderne europäische Ostpolitik - das heißt heute: Heranführung der Ukraine an die EU, Partnerschaft mit Russland, Demokratisierung von Belarus, Kooperation mit Zentralasien und ein gedeihliches Miteinander am Schwarzen Meer und im Kaukasus. Das heißt auch: enge Zusammenarbeit mit den USA und unseren anderen Verbündeten, um diese gemeinsamen Ziele zu verwirklichen. Das heißt vor allem: ein gemeinsamer Raum des Friedens und des Wohlstands von der Atlantikküste bis nach Sibirien oder noch weiter gefasst: von Vancouver bis nach Wladiwostok."

Die größte Unterstützung erhielt die Östliche Partnerschaft aus dem Bundeskanzleramt. Die Bundeskanzlerin selbst, die als einzige Vertreterin der europäischen "Schwergewichte» beim Prager Gipfel im Mai 2009 anwesend war, gab dem polnisch-schwedischen Vorschlag ihre volle Unterstützung: "Die Östliche Partnerschaft ist ein Projekt, das dem Frieden, der Entwicklung und dem Austausch dient«, definierte Bundeskanzlerin Merkel die neue Strategie der EU für die Nachbarstaaten im Osten Europas. Im Bundestag selbst hat die Östliche Partnerschaft eine starke Unterstützung erhalten. Parallel dazu änderte sich die Wahrnehmung Russlands in Deutschland. Die Auswertung der Nachrichtenmedien beweist, dass im Laufe der letzten Jahre die Sicht auf die Transformationsprozesse im Osten Europas vielschichtiger geworden ist. Dies verändert auch die Sicht der Politik auf Russland und den postsowjetischen Raum. Anfang des Jahrzehnts trat der russische Präsident Vladimir Putin noch im Bundestag auf, heute überwiegt eine kritische Betrachtungsweise seiner Präsidentschaft.

\section{Gemeinsame Interessen im Osten Europas}

Die Politik Deutschlands gegenüber den Nachbarstaaten im Osten spielt sich auf zwei Ebenen ab. Einerseits bemüht sich die Bundesregierung um gute Beziehungen zu Russland, d. h. eine Neuauflage des Partnerschafts- und Kooperationsabkommens (PKA) zwischen der EU und Russland. Andererseits wurden die Bezie- hungen zu den anderen Nachbarstaaten in der Strategie der Östlichen Partnerschaft der EU neu definiert. Bei der Gestaltung dieser beiden Richtungen der europäischen Ostpolitik spielt neben der Bundesrepublik Deutschland Polen eine Schlüsselrolle, und zwar nicht nur wegen seiner inhaltlichen Kompetenz und staatlichen Ressourcen, die dafür aufgewandt werden müssen, sondern auch wegen des bestehenden Konsenses in der politischen Elite des Landes. Sowohl bei der Verschiebung der Verhandlungen um ein neues PKA mit Russland Ende 2006 als auch bei der Gestaltung der Östlichen Partnerschaft 2008 ist dies deutlich zum Ausdruck gekommen. Außenminister Sikorski, der als Architekt der Östlichen Partnerschaft gilt, hatte immer betont, dass diese nicht gegen Russland gerichtet ist.

Die veränderte deutsche Wahrnehmung der Entwicklung in Russland wurde in Polen mit Genugtuung zur Kenntnis genommen, insbesondere der Auftritt der Bundeskanzlerin in Tiflis im August 2008 nach der Beendigung der militärischen Auseinandersetzungen in Georgien. Die Veränderung des Russlandbildes in Deutschland und eine gewisse "Arbeitsteilung « bei der Gestaltung der EU-Ostpolitik war ein wichtiges Signal für die Wiederbelebung der deutsch-polnischen Kooperation. Nach dem Regierungswechsel in Warschau im Herbst 2007 entspannte sich die Lage in den bilateralen Beziehungen; nach den historisch kontroversen Themen konnte die Hinwendung zum Thema Ostpolitik die Kooperation beleben. Hinzu kam die Tatsache, dass es Polen gelang, trotz der deutschen Zurückhaltung die schwedische Diplomatie für die Östliche Partnerschaft zu gewinnen und auf diese Weise die Einteilung in alte und neue Mitglieder in der EU zu überwinden. Es zählte auch, dass Polen half, die Litauer im Frühjahr 2008 zu überzeugen, die Blockade des Verhandlungsmandats für das Partnerschafts- und Kooperationsabkommen mit Russland aufzugeben. Der Deal war perfekt, denn er war einerseits ein Gegengewicht zur Mittelmeer-Union und andererseits ein Mandat für die EUKommission für die PKA-Verhandlungen mit Russland. Die EU-Außenminister konnten am 26. April 2008 den Vorschlag Polens für die Östliche Partnerschaft annehmen und die EU-Kommission beauftragen, die Verhandlungen mit Russland über ein neues Partnerschafts- und Kooperationsabkommen aufzunehmen.

Nach der Bundestagswahl im September 2009 übernahm die FDP mit Guido Westerwelle das Auswärtige Amt. Die erste Auslandsreise des neuen Außenministers führte am 30. Oktober 2009 nach Warschau. Während des Besuches wurde die Wiederbelebung des Weimarer Dreiecks vereinbart, einer deutsch-polnisch-franzö- 


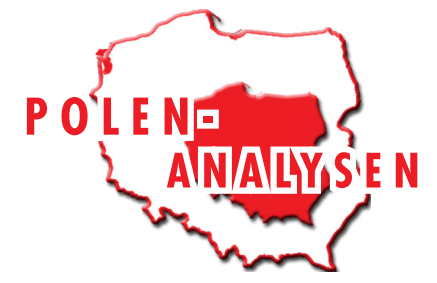

polen-analysen $72 / 10$

sischen Initiative aus dem Jahr 1991, die einer Koordinierung der Europapolitik der drei EU-Staaten dienen soll. Als neue Herausforderung des Weimarer Dreiecks gilt die europäische Ostpolitik.

Bei der Analyse der gemeinsamen Interessen im Osten Europas dürfen die klaren Interessen der deutschen Wirtschaft, die vom Ostausschuss der Deutschen Wirtschaft deutlich vertreten werden, nicht außer Acht gelassen werden. Russland ist einer der wichtigsten Absatzmärkte für die deutsche Industrie, insbesondere die Automobil- und Maschinenindustrie. Im Jahr 2008 erreichte der Warenaustausch mit Russland über 68 Milliarden Euro, und der gesamte Osthandel Deutschlands 84 Milliarden Euro. Schon heute werden 50 Prozent des russischen Handels mit der EU abgewickelt. 80 Prozent der russischen Energieexporte gehen in die EU; über 75 Prozent der ausländischen Investitionen kommen aus der EU. Deutschland steht bei den russischen Exporten und Importen an erster Stelle. Die Wirtschaftskrise veränderte jedoch das Kräfteverhältnis grundlegend. Angesichts des schrumpfenden Exports nach Russland reduzierte sich der Druck der exportierenden Wirtschaft auf die Regierung, gute Beziehungen nach Russland aufrechtzuerhalten.

Immer wieder sorgen auch negative Schlagzeilen aus Russland für Verstimmung. In diesem Kontext muss auch erwähnt werden, dass im vergangenen Jahr der deutschpolnische Warenaustausch (Exporte und Importe) höher war als der deutsch-russische Handel. Polen und Russland kämpfen seit Jahren um den ersten Platz in der Statistik. Dabei sticht die unterschiedliche Struktur der Warenströme und Akteure ins Auge. Im Handel mit Polen sind es vor allem kleine und mittlere Unternehmen mit hochwertiger Produktion, im Handel mit Russland sind es große Firmen aus der Automobil- und Maschinenindustrie, die eine bessere Lobby haben. Bei den Importen sind sowohl Deutschland als auch Polen auf die Energierohstoffe aus Russland angewiesen, mit dem Unterschied, dass dieses Thema in Polen politisch instrumentalisierbar ist und in Deutschland (noch) nicht.

\section{Implementierung der Östlichen Partnerschaft}

Nach dem EU-Gipfel im Prag ist es gelungen, eine Reihe von Schritten zu unternehmen, die der Östlichen Partnerschaft ein konkretes Gesicht verleihen. Zunächst wurden in der EU-Kommission die Rahmenbedingungen für die Finanzierung festgelegt. Im nächsten Schritt konstituierte sich das zivilgesellschaftliche Forum, welches die Aktivitäten der Nichtregierungsorganisationen in den Partnerstaaten koordiniert. Ein erstes Treffen fand im November 2009 in Brüssel statt; das nächste ist für November 2010 in Berlin anberaumt. Auf diesem Feld liegt eine große Chance für die Östliche Partnerschaft der EU - weg von der großen Politik hin zum einzelnen Bürger, der die Reformen von unten antreibt. $\mathrm{Da}$ es sich um eine Region handelt, die sicherlich in den nächsten Jahren nicht der EU beitreten wird, ist die gesellschaftliche Dimension das Wichtigste in dieser Strategie und muss es hier um eine langfristige Einbindung der Menschen und Staaten der Östlichen Partnerschaft in paneuropäische Projekte, Diskussionen und Prozesse gehen. Dabei handelt es sich um einen langfristigen Prozess. Die Östliche Partnerschaft soll den Menschen vermitteln, dass sie der europäischen Familie angehören, wenn auch ohne Mitgliedschaft in der EU. Die Bildung des »Freundeskreises der Östlichen Partnerschaft« während des letzen Außenministertreffens im Mai in Zoppot eröffnet Partnern von außerhalb der EU die Möglichkeit, sich zu beteiligen. Dies werden sicherlich auch US-amerikanische NGOs und Stiftungen nutzen, die von Anfang an die Östliche Partnerschaft als Unterstützung der Zivilgesellschaft im Osten Europas interpretiert haben.

In den Partnerstaaten entwickelte sich allerdings die politische Lage nach der Verabschiedung der Östlichen Partnerschaft in Prag nicht im Sinne der EU. Zunächst erwies die Ukraine der neuen EU-Strategie einen Bärendienst, indem sie noch vor dem Prager Gipfel die Strategie heftig kritisierte und ablehnte. In Berlin wurde die kritische Haltung der Ukraine zuungunsten Warschaus ausgelegt. Es wurde klar, dass Warschau den Partnerstaaten die Strategie nicht angemessen erklärt hatte. In Warschau dagegen ist Teilen der politischen Eliten der Geduldsfaden gerissen und eine kritische Betrachtung der Entwicklung trat in den Vordergrund. Bei einem gemeinsamen Besuch der Außenminister Steinmeier und Sikorski in Kiew kam es zu keiner Klärung des Problems. Im Sommer 2009 legte die Kampagne für die Präsidentschaftswahl die Ukraine lahm; die Wahl hat die Lage zuungunsten der EU verändert. Nach der Wahl ist der neu gewählte Präsident Wiktor Janukowitsch nach Brüssel gefahren und hat ein Zeichen für die Beziehungen zur EU gesetzt. Die folgende Entwicklung hat jedoch das Fundament für eine erfolgreiche Kooperation der EU mit der Ukraine in Frage gestellt: Der Vertrag über die Stationierung der Schwarzmeerflotte in Sewastopol auf der Krim und über russische Gaslieferungen, der am 21. April 2010 von den Präsidenten Dimitri Medwedew und Janukowitsch unterzeichnet wurde, lockert die EU-Anbindung der Ukraine. Der Vertrag sieht vor, dass die vor dem Staatsbankrott stehende Ukraine durch Veränderungen der Gasverträge 


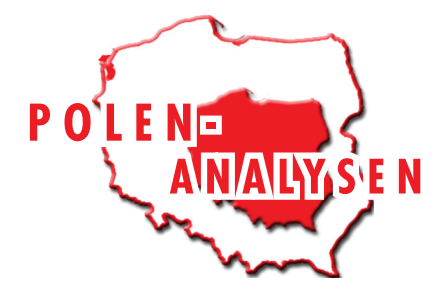

von Russland einen Rabatt von umgerechnet 30 Milliarden Euro erhält. Als Gegenleistung darf die russische Schwarzmeerflotte mindestens bis 2042 auf der ukrainischen Halbinsel Krim stationiert bleiben. In Polen sorgten schon vor den ukrainischen Präsidentschaftswahlen die Auseinandersetzungen innerhalb des »orangen Lagers» für große Irritationen. Im Zusammenhang mit der polnisch-russischen Annäherung ist mit einer nachlassenden Unterstützung der polnischen Eliten für den europäischen Weg der Ukraine zu rechnen.

In Belarus sieht die Lage genauso schlecht aus. Der Verlauf der Kommunalwahlen im April 2010 lässt vermuten, dass die von der EU erhoffte Demokratisierung in diesem Land noch lange auf sich warten lassen wird. Die Öffnung Minsks macht keine Fortschritte. Die Spannungen zwischen dem belarussischen Präsidenten Alexander Lukaschenko und Moskau werden wahrscheinlich dafür sorgen, dass sich auch in den nächsten Monaten das Verhältnis zwischen der EU und Belarus nicht vollständig abkühlen wird, ein Durchbruch ist jedoch nicht zu erwarten. Auch in den anderen Partnerstaaten der Östlichen Nachbarschaft gibt es keine positiven Entwicklungen. Der Dialog zwischen Armenien und der Türkei steckt fest und Fortschritte bei der Demokratisierung des Landes sind nicht zu erkennen. Aserbaidschans Interesse an der EU sinkt mit jedem neuen Problem beim Bau der Nabucco-Pipeline. Die Entscheidung, die Erschließung der Gasfelder für die NabuccoPipeline auf das Jahr 2017 zu verschieben, trägt auch nicht dazu bei, die Beziehungen zu intensivieren. Einzig in Moldawien gibt es eine Regierung, die ernsthaft einen Weg Richtung EU anstrebt.

\section{Fazit}

Die bilateralen Beziehungen zwischen Polen und Russland spielen eine Schlüsselrolle für die künftige Gestaltung des Verhältnisses der EU zu Russland. Vor diesem Hintergrund hat sich Deutschland in den vergangenen Jahren stets um eine positive Haltung gegenüber Russland bemüht und zugleich eine ausbalancierte Ostpolitik der EU verfolgt. Der prorussische deutsche Vorschlag »Annäherung durch Verflechtung « des damaligen Außenministers Steinmeier, der von Bundeskanzlerin Merkel akzeptiert wurde, konnte von der polnisch-schwedischen Initiative der Östlichen Partnerschaft ergänzt werden. Bundeskanzlerin Merkel unterstützte dies durch ihre Anwesenheit beim Gründungsgipfel in Prag.

Die ausgewogene und pragmatische Russland-Politik der polnischen Regierung unter Ministerpräsident Donald Tusk, die zusammen mit anderen Faktoren zu einer Erwärmung der polnisch-russischen Beziehungen führte, wurde von der deutschen Seite unterstützt. Der Wendepunkt war in diesem Falle der Besuch des russischen Ministerpräsidenten Putin bei der Gedenkfeier zum 70. Jahrestag des Beginns des Zweiten Weltkriegs am 1. September 2009 auf der Westerplatte. Diese Entwicklung stärkte die Position Polens bei der Gestaltung der europäischen Ostpolitik. Die Katastrophe in Smolensk am 10. April 2010, bei der neben dem polnischen Staatspräsidenten Lech Kaczyński weitere 95 Vertreter der polnischen Politik und des öffentlichen Lebens tödlich verunglückten, und die anschließende polnisch-russische Annäherung können eine weitere Chance für die Verbesserung der Beziehungen Polens und der gesamten EU zu Russland darstellen.

Auch im Rahmen des Weimarer Dreiecks kann die EU-Ostpolitik konzeptionell weiterentwickelt werden. Beim letzten Treffen der Außenminister des Weimarer Dreiecks am 27. April 2010 in Bonn nahm der ukrainische Außenminister Konstantin Hryschtschenko als Gast teil. Außenminister Westerwelle begrüßte übereinstimmend mit seinem polnischen Amtskollegen den offiziell bekundeten Willen der neuen ukrainischen Führung, an den europäischen Werten und dem Annäherungskurs in Richtung EU festzuhalten, und sagte weitere Unterstützung bei der Umsetzung der ehrgeizigen ukrainischen Reformagenda sowie der Verbesserung der Beziehungen zu Russland zu. Beim nächsten Außenministertreffen in Paris am 23. Juni soll der russische Außenminister Sergej Lawrow dabei sein. Die bisherige erfolgreiche Arbeit im Dreieck Berlin-Warschau-Moskau soll hier für das Weimarer Dreieck genutzt werden. Ob dies gelingt, bleibt abzuwarten.

Das gegenwärtig geringe Interesse der EU-Akteure an der Strategie der Östlichen Partnerschaft und die negativen Entwicklungen in den Partnerstaaten selbst bedeuten nicht das Ende der Östlichen Partnerschaft. Die Strategie muss geändert werden. Der Umbau muss eine Verstärkung der zivilgesellschaftlichen Aktivitäten zur Folge haben und weniger offizielle Begegnungen und Projekte. Auf diese Weise kann die Östliche Partnerschaft eine Unterstützung der Transformationsprozesse in den Gesellschaften der östlichen Nachbarstaaten der EU gewährleisten. Das beste Beispiel für den Strategiewechsel ist die Gründung der »Gruppe der Freunde der Östlichen Partnerschaft«. Diese soll »allen dritten Staaten, die sich an einzelnen Projekten der Partnerschaft beteiligen möchten, als Anlaufstelle und informelles Forum dienen".

Unter dem Eindruck der gegenwärtigen Entwicklung im Osten Europas kann es nicht verwundern, dass Russland doch wieder im Vordergrund der deutschen Osteuropapolitik steht, wie die Stellungnahme des Staatsmi- 


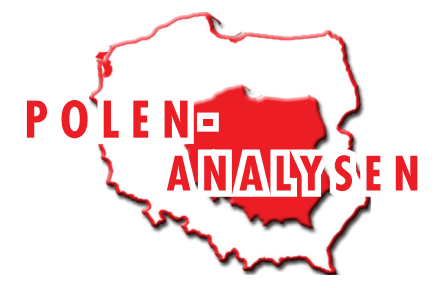

nisters Hoyer in einem Namensartikel für die Frankfurter Allgemeine Zeitung im April zeigt. Die SPD-Fraktion mit dem ehemaligen Außenminister Steinmeier als Vorsitzenden bestätigte ebenfalls die Bedeutung Russlands für die deutsche Osteuropapolitik in einem Antrag der SPDFraktion vom 23. März 2010 mit dem Titel »Modernisierungspartnerschaft mit Russland - Gemeinsame Sicherheit in Europa durch stärkere Kooperation und Verflechtung . Bedauerlich ist, dass der EU - Russland Gipfel, der am 1. Juni 2010 in Rostow/Don stattfand, ohne konkrete Ergebnisse blieb. Russland forderte die Aufhebung der Visumspflicht, die EU dagegen bat um eine eindeutige Positionierung Russlands in Sachen WTO-Beitritt und Handelspolitik. Bisher ist die Haltung Russlands hier zweideutig. Einerseits kündigte Präsident Medwedew den WTO-Beitritt bis Ende des Jahres an, andererseits treibt Ministerpräsident Putin die Zollunion mit Kasachstan und Belarus voran, die einen Beitritt verzögert, wenn nicht sogar verhindert. Die WTO-Mitgliedschaft Russlands wird aber als Schlüsselfrage des neuen EU-Russland-Abkommens betrachtet. Ohne diese Mitgliedschaft müssen alle Einzelfragen der Handelspolitik in entsprechenden Verträgen geregelt werden. Im Vorfeld des Gipfels wurde viel von einer »Modernisierungspartnerschaft" geredet, die Außenminister Westerwelle und Lawrow sogar in einem gemeinsamen Artikel in der deutschen und russischen Presse darstellten.

Schließlich sei auf den Besuch des russischen Präsidenten Medwedew bei Bundeskanzlerin Merkel am 4./5. Juni 2010 hingewiesen, dessen Ergebnis eine Reihe von neuen Kooperationsvorschlägen im sicherheitspolitischen Bereich sind, die für die deutsch-polnischen Beziehungen und die europäische Ostpolitik von hoher Bedeutung sind. Deutschland und Russland haben eine engere Zusammenarbeit zwischen der EU und Moskau im Bereich der Sicherheit vorgeschlagen. Konkret nannten Merkel und Medwedew die Gründung eines Gemeinsamen Politik- und Sicherheitskomitees. Dem Gremium sollen die EU-Außenbeauftragte Catherine Ashton und der russische Außenminister Lawrow angehören. In diplomatischen Kreisen wurde der Vorschlag als Kompromiss interpretiert, denn im Gegenzug hatte sich Medwedew bereit erklärt, den Krisenherd Transnistrien auf die Agenda zu setzen. Das Thema soll zunächst im Rahmen der bestehenden "5+2 Gruppe» (Russland, Ukraine, Moldawien, Transnistrien, OSZE, EU und USA) behandelt werden. Merkel wies darauf hin, dass es sich in diesem Fall um ein Problem unmittelbar vor den EU-Außengrenzen handelt. Unter diesem Aspekt muss der Besuch des moldawischen Ministerpräsidenten Vladimir Filat bei der Bundeskanzlerin im Mai 2010 gesehen werden. Die polnische Seite ist sicherlich mit diesem Kompromiss einverstanden, setzte sich die polnische Regierung in den vergangenen Jahren doch für ein stärkeres Engagement der EU in Moldawien ein. »Insgesamt sollten beide Seiten, d. h. EU und Russland, bei der Lösung von Konflikten und bei der zivilen und militärischen Krisenbewältigung enger zusammenrücken«, so Merkel bei der Pressekonferenz.

Hinzu kommen Vorschläge im innenpolitisch heiklen Bereich der Visapolitik. Zu den Wünschen Russlands nach Visafreiheit für die EU sagte Merkel Medwedew zu, sich in dieser Frage für Russland einzusetzen. Die Visapolitik ist jedoch eine Angelegenheit, die im Rahmen des Schengenraumes behandelt werden muss. Es ist kaum möglich, diese Initiative ohne Verknüpfung mit den Staaten der Östlichen Partnerschaft zu lösen. Die ersten Reaktionen aus Warschau weisen eindeutig in diese Richtung.

Es ist der beste Beweis für die Verbesserung der deutsch-polnischen Beziehungen, wenn eine deutschrussische Initiative in Polen mit Interesse zur Kenntnis genommen wird. In negativer Erinnerung sind immer noch die Ostseepipeline und die Verbindungen zwischen dem damaligen Bundeskanzler Gerhard Schröder und Staatspräsident Putin. Durch die Absage der deutschpolnischen Regierungskonsultationen Anfang Juni verpasste Ministerpräsident Tusk jedoch die Chance, von der Bundeskanzlerin persönlich über die bevorstehenden Konsultationen mit Medwedew informiert zu werden. Hinzu kommt, dass sich Polen gerade im Präsidentschaftswahlkampf befindet und außerdem eine Hochwasserkatastrophe zu bewältigen hat. Schließlich konzentriert sich die polnische Europapolitik derzeit auf die Aufrechterhaltung des Einflusses in der EU-Finanz- und Wirtschaftspolitik. Aufgrund der Tatsache, dass Polen noch nicht der Eurozone beitreten konnte, rufen die Reformvorschläge der Euro-Finanzminister in Polen die Angst vor einem Europa unterschiedlicher Geschwindigkeiten hervor. Die kritischen Äußerungen des neuen Präsidenten der Polnischen Nationalbank (Bank Narodowy Polski - BNP), des ehemaligen Ministerpräsidenten Marek Belka, über den Zustand des Euro sind nicht besonders hilfreich für einen künftigen Beitritt zur Eurozone. Der Besuch von Regierungschef Tusk und der Mehrheit der polnischen Regierungsmitglieder in Brüssel wird sicherlich die interne Entwicklung der EU nicht aufhalten können. Zum ersten Mal in der Geschichte argumentiert jedoch Polen auf wirtschaftlicher Basis und befreit sich von den Ängsten gegenüber seinen Nachbarn. Dies ist das Polen des 21. Jahrhunderts.

(Informationen über den Autor und Lesetipps finden Sie auf der nächsten Seite) 


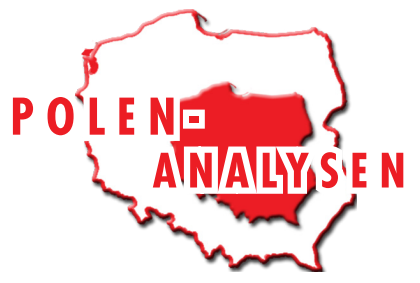

Über den Autor

Cornelius Ochmann ist Osteuropa-Experte der Bertelsmann Stiftung. Sein Arbeitsschwerpunkt liegt auf der Ostpolitik der Europäischen Union unter besonderer Berücksichtigung der Beziehungen zwischen der EU und Russland.

\section{Lesetipps:}

- Cornelius Ochmann: Im Osten was Neues; spotlight europe 2009/06 http://www.bertelsmann-stiftung.de/cps/rde/ xbcr/SID-5047C3D3-4D7E8FO/bst/Deutsch_spotlight_Im\%20Osten\%20was\%20Neves_09-05-28.pdf

- Cornelius Ochmann (Co-Autor) mit Joachim Fritz-Vannahme, Armando García Schmidt, Margarethe Gawelek und Christian-Peter Hanelt: Hallo Nachbar! Für eine neue EU-Politik von Marokko bis Aserbaidschan; spotlight europe 2008/07 http://www.bertelsmann-stiftung.de/cps/rde/xbcr/SID-165E5E1E-2055C247/bst/spotlight_Nach bar_07_2008_.pdf

\section{Tabellen und Grafiken}

\section{Der Außenhandel Polens nach Ziel- und Herkunftsländern, Januar-September 2009}

\begin{tabular}{|c|c|c|c|c|c|c|c|c|}
\hline & \multirow{2}{*}{$\begin{array}{l}\text { Export } \\
\text { (Mio. } \\
\text { Euro) }\end{array}$} & \multirow{2}{*}{$\begin{array}{l}\text { Import } \\
\text { (Mio. } \\
\text { Euro) }\end{array}$} & \multicolumn{2}{|c|}{ Dynamik (\%) } & \multirow{2}{*}{$\begin{array}{c}\begin{array}{c}\text { Anteil } \\
\text { Export } \\
(\%)\end{array} \\
\text { Jan.-Sept. } \\
2009\end{array}$} & \multirow{2}{*}{$\begin{array}{c}\begin{array}{c}\text { Anteil } \\
\text { Export } \\
(\%)\end{array} \\
\text { Jan.-Sept. } \\
2008\end{array}$} & \multirow{2}{*}{$\begin{array}{c}\begin{array}{c}\text { Anteil } \\
\text { Import } \\
(\%)\end{array} \\
\text { Jan.-Sept. } \\
2009\end{array}$} & \multirow{2}{*}{$\begin{array}{c}\begin{array}{c}\text { Anteil } \\
\text { Import } \\
(\%)\end{array} \\
\text { Jan.-Sept. } \\
2008\end{array}$} \\
\hline & & & Export & Import & & & & \\
\hline $\begin{array}{l}\text { Handelsumsatz Polen } \\
\text { insgesamt }\end{array}$ & $69.997,4$ & $76.379,8$ & 78,4 & 70,5 & 100,0 & 100,0 & 100,0 & 100,0 \\
\hline $\begin{array}{l}\text { wirtschaftlich ent- } \\
\text { wickelte Länder, darin: }\end{array}$ & $59.714,2$ & $52.523,0$ & 80,7 & 69,5 & 85,3 & 82,9 & 68,8 & 69,8 \\
\hline $\begin{array}{l}\text { Länder außerhalb der } \\
\text { EU }\end{array}$ & $4.215,5$ & $5.631,4$ & 92,4 & 70,9 & 6,0 & 5,1 & 7,4 & 7,3 \\
\hline $\begin{array}{l}\text { EU-Mitgliedsländer, } \\
\text { darin: }\end{array}$ & $55.498,7$ & $46.891,6$ & 79,9 & 69,3 & 79,3 & 77,8 & 61,4 & 62,5 \\
\hline Deutschland & $18.415,8$ & $17.220,7$ & 82,3 & 67,7 & 26,3 & 25,1 & 22,5 & 23,5 \\
\hline andere Länder, darin: & $10.283,2$ & $23.856,9$ & 67,2 & 73,0 & 14,7 & 17,1 & 31,2 & 30,2 \\
\hline $\begin{array}{l}\text { Gemeinschaft Un- } \\
\text { abhängiger Staaten } \\
\text { (GUS) }\end{array}$ & $5.438,4$ & $7.920,9$ & 57,8 & 58,2 & 7,8 & 10,5 & 10,4 & 12,6 \\
\hline Russland & $2.586,5$ & $6.619,3$ & 55,2 & 62,1 & 3,7 & 5,2 & 8,7 & 9,8 \\
\hline Ukraine & $1.786,7$ & 541,8 & 51,2 & 43,5 & 2,6 & 3,9 & 0,7 & 1,2 \\
\hline Belarus & 638,0 & 433,3 & 80,6 & 60,4 & 0,9 & 0,9 & 0,6 & 0,7 \\
\hline
\end{tabular}

Quelle: Ministerstwo Gospodarki [Ministerium für Wirtschaft]: Sytuacja w handlu zagranicznym po 3 kwartalach 2009r. [Die Situation des Außenhandels im 1.-3. Quartal 2009]. Warszawa, 30.11. 2009. http://www.mg.gov.pl/NR/rdonlyres/2556BC8A-2D6D-473DAFF6-7E IA6ABE7798/58398/Ocenahzpo3kw2009r.pdf (abgerufen am 11.06. 2010). 


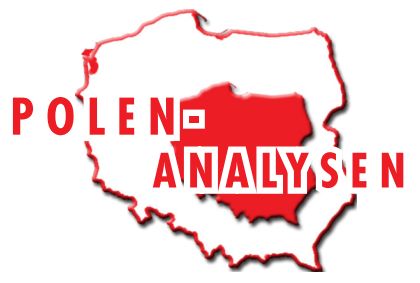

Zielländer des Exports aus Polen Januar-September 2009. Prozentualer Anteil einzelner Ländergruppen und Länder

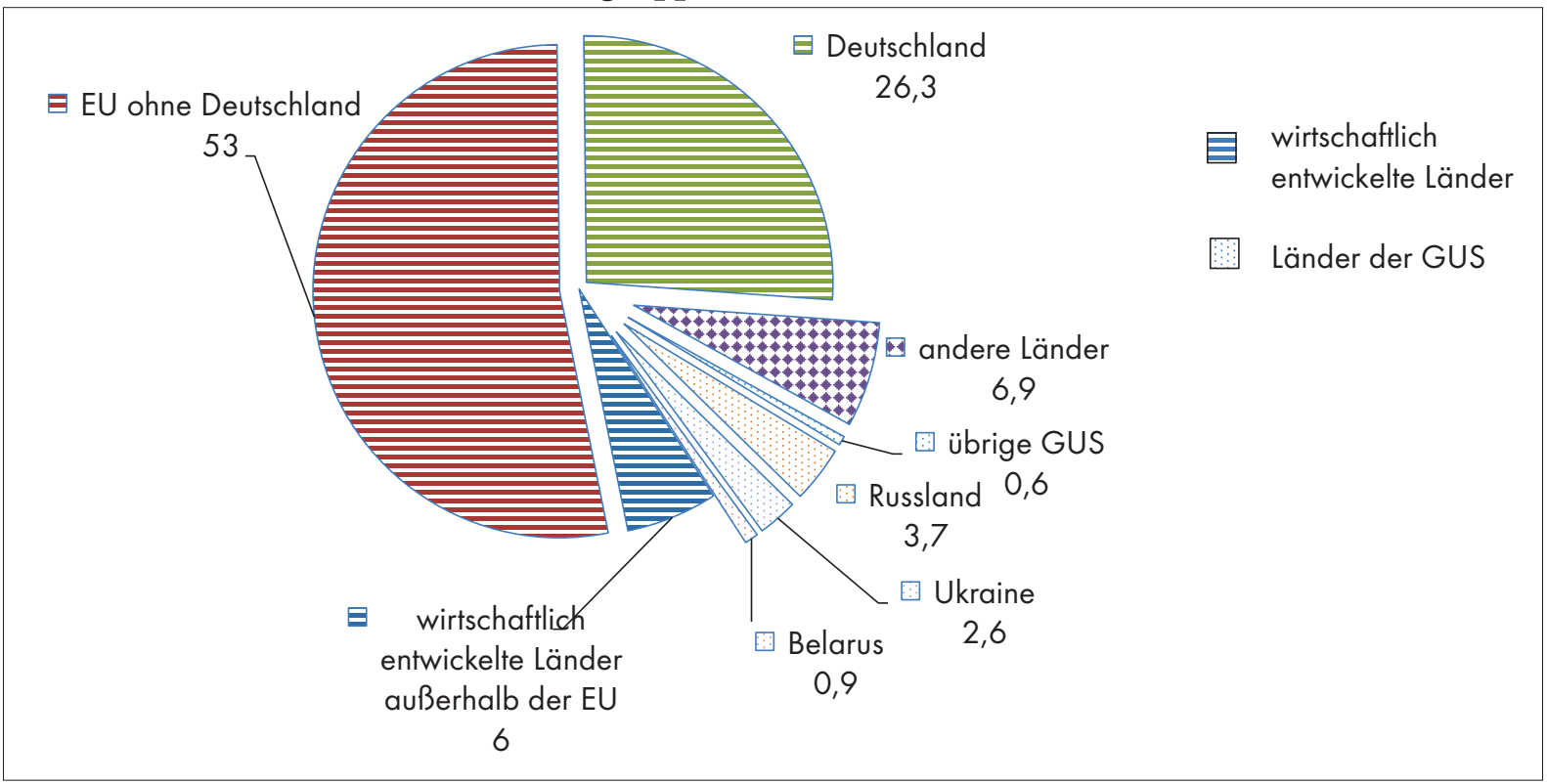

Zielländer des Exports aus Polen Januar-September 2008.

Prozentualer Anteil einzelner Ländergruppen und Länder

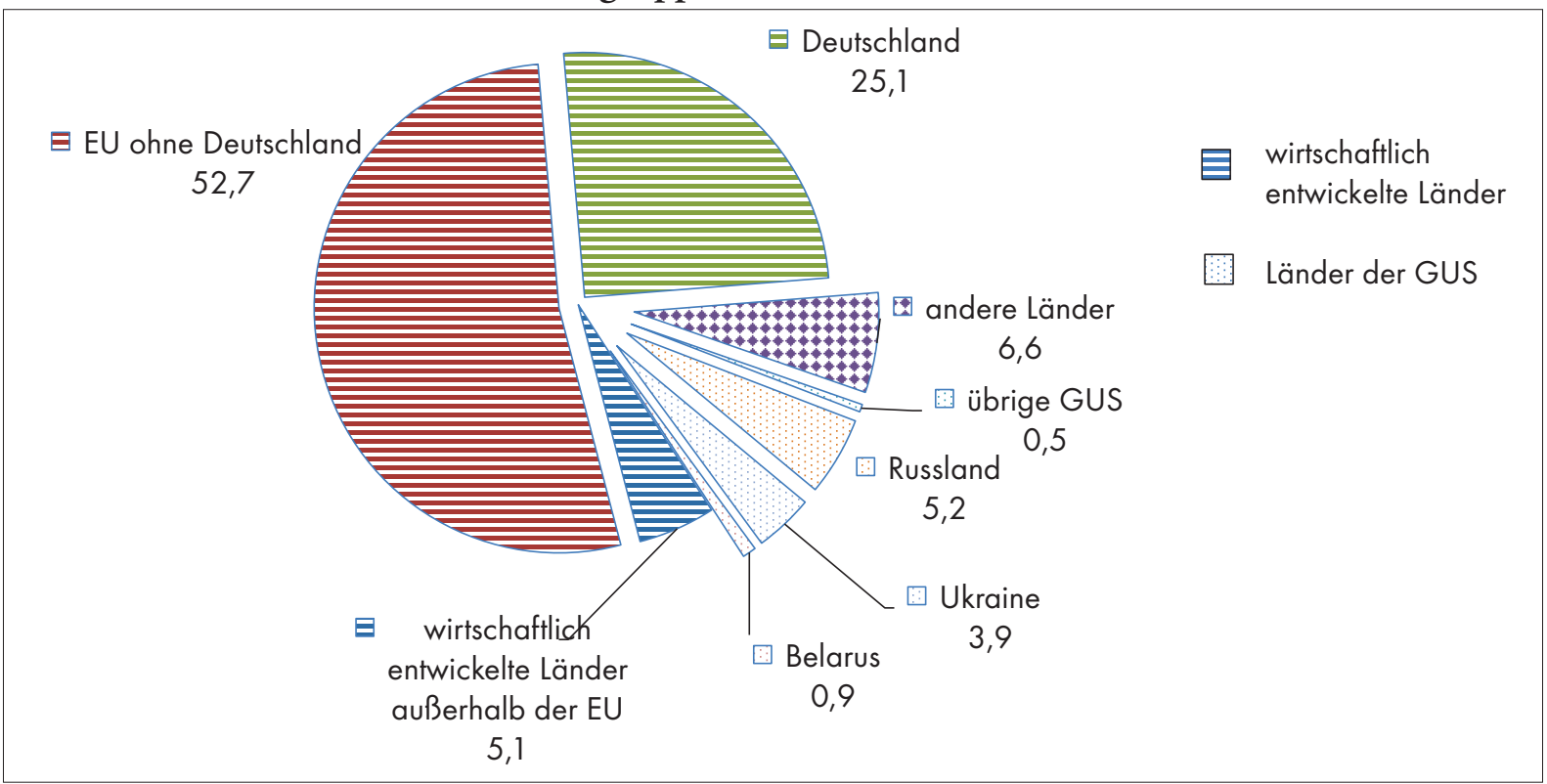

Quelle: Ministerstwo Gospodarki [Ministerium für Wirtschaft]: Sytuacja w handlu zagranicznym po 3 kwartalach 2009r. [Die Situation des Außenhandels im 1.-3. Quartal 2009]. Warszawa, 30.11. 2009. http://www.mg.gov.pl/NR/rdonlyres/2556BC8A-2D6D-473DAFF6-7E IA6ABE7798/58398/Ocenahzpo3kw2009r.pdf (abgerufen am 11.06. 2010). 


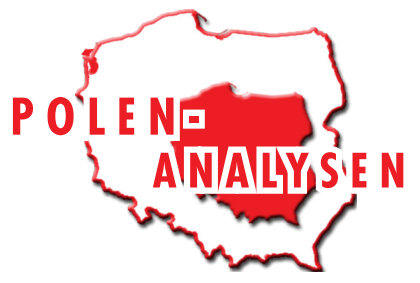

Herkunftsländer des Imports nach Polen Januar-September 2009.

Prozentualer Anteil einzelner Ländergruppen und Länder

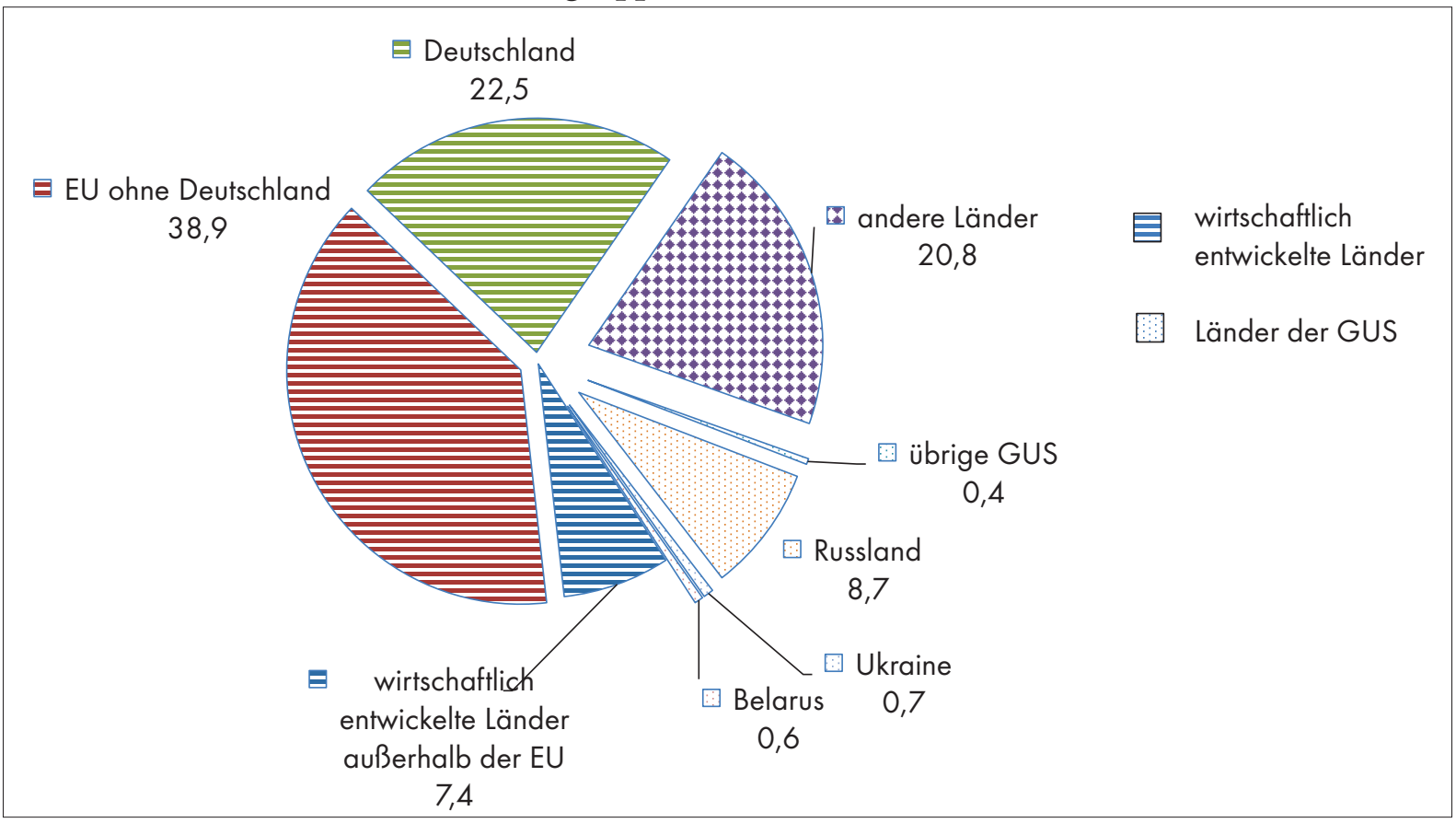

Herkunftsländer des Imports nach Polen Januar-September 2008.

Prozentualer Anteil einzelner Ländergruppen und Länder

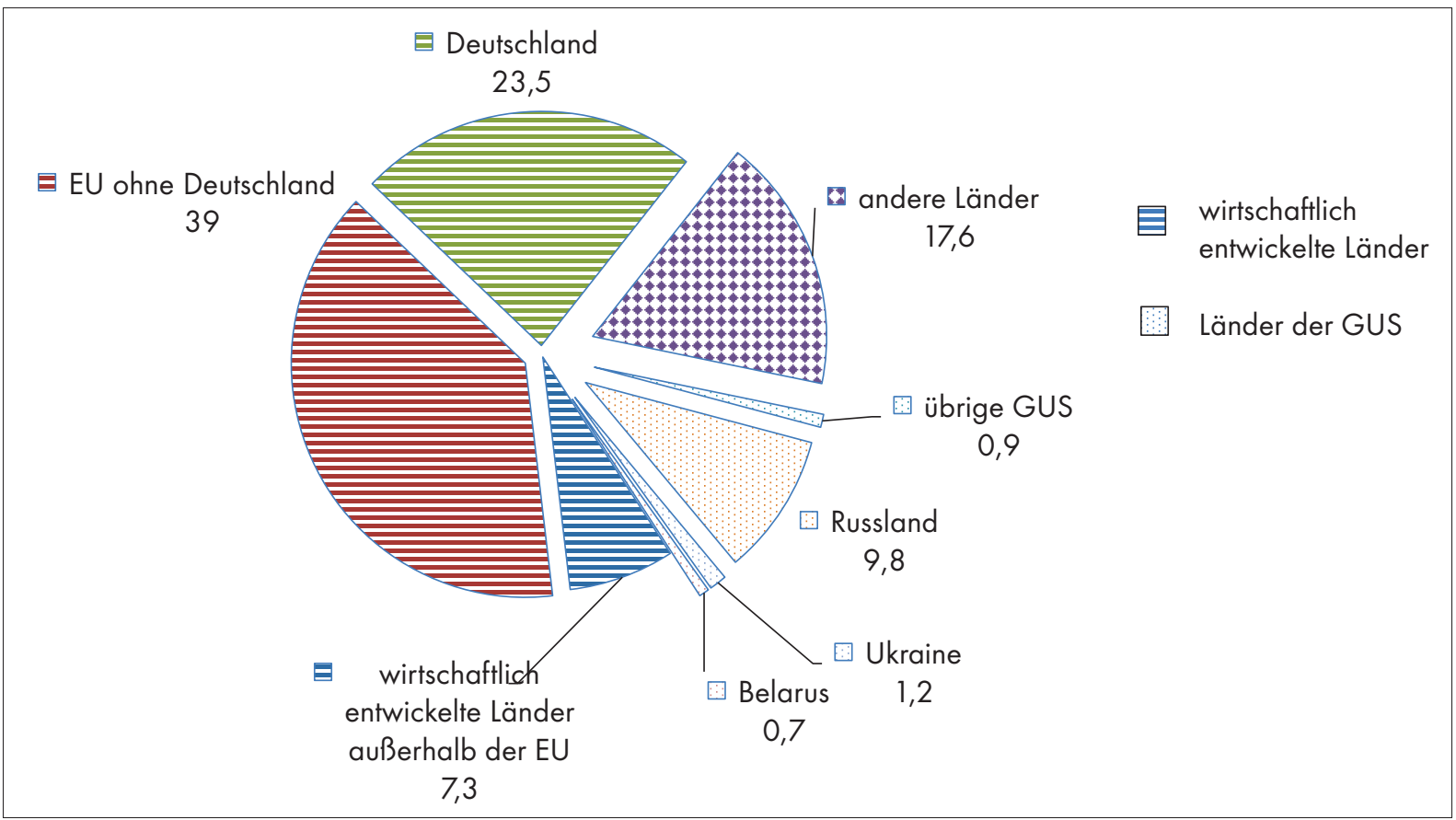

Quelle: Ministerstwo Gospodarki [Ministerium für Wirtschaft]: Sytuacja w handlu zagranicznym po 3 kwartalach 2009r. [Die Situation des Außenhandels im 1.-3. Quartal 2009]. Warszawa, 30.11. 2009. http://www.mg.gov.pl/NR/rdonlyres/2556BC8A-2D6D-473DAFF6-7E IA6ABE7798/58398/Ocenahzpo3kw2009r.pdf (abgerufen am 11.06. 2010). 


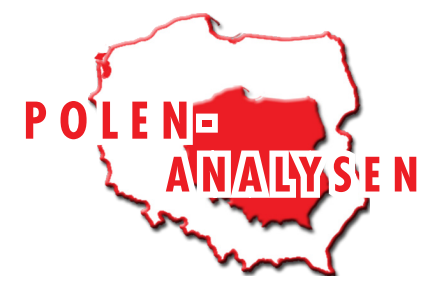

\section{Sympathie- und Antipathiewerte gegenüber ausgewählten Nationen}

Veränderungen der Sympathiewerte gegenüber ausgewählten Nationen (\%)

\begin{tabular}{|l|c|c|c|}
\hline & $\mathbf{1 9 9 3}$ & $\mathbf{2 0 0 1}$ & $\mathbf{2 0 1 0}$ \\
\hline Armenien & keine Angabe & keine Angabe & 29 \\
\hline Belarus & 19 & 26 & 34 \\
\hline Deutschland & 23 & 32 & 51 \\
\hline Frankreich & 61 & 55 & 32 \\
\hline Georgien & keine Angabe & keine Angabe & 50 \\
\hline Großbritannien & 47 & 44 & 34 \\
\hline Russland & 17 & 23 & 34 \\
\hline Ukraine & 12 & 19 & 45 \\
\hline Vereinigte Staaten von Amerika & 62 & 50 & 0.19 \\
\hline
\end{tabular}

CBOS, BS/12/2010: Stosunek Polaków do innych narodów [Das Verhältnis der Polen zu anderen Nationen]. Warszawa, 01/2010. www. cbos.pl

Veränderungen der Sympathiewerte gegenüber ausgewählten Nationen (\%)

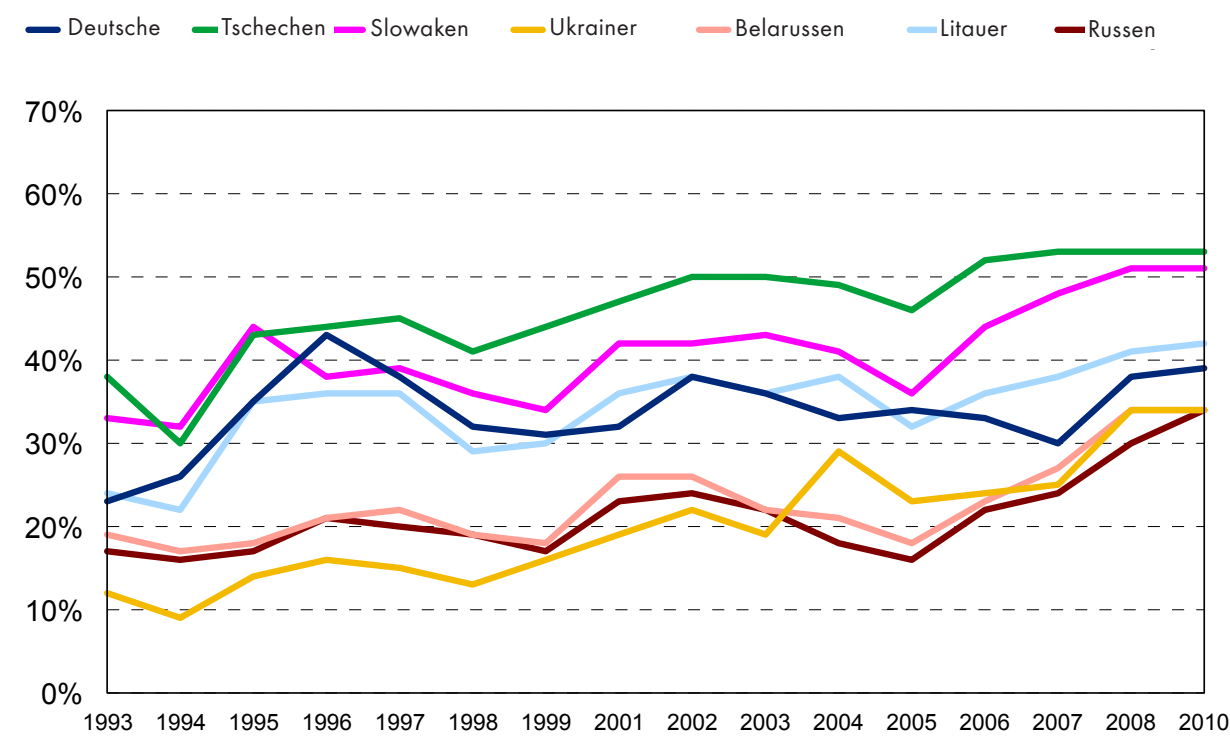

CBOS, BS/12/2010: Stosunek Polaków do innych narodów [Das Verhältnis der Polen zu anderen Nationen]. Warszawa, 01/2010. www.cbos.pl 


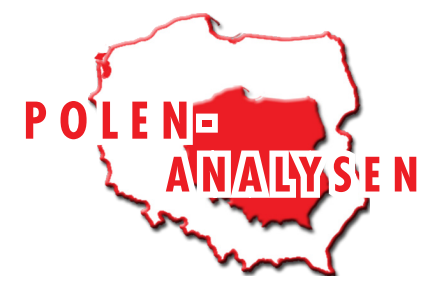

Veränderungen der Antipathiewerte gegenüber ausgewählten Nationen (\%)

\begin{tabular}{|l|c|c|c|}
\hline & 1993 & 2001 & 2010 \\
\hline Armenien & keine Angabe & keine Angabe & 23 \\
\hline Belarus & 47 & 40 & 23 \\
\hline Frankreich & 53 & 36 & 10 \\
Georgien & 9 & 9 & 22 \\
\hline Großbritannien & keine Angabe & keine Angabe & 12 \\
\hline Russland & 16 & 18 & 31 \\
\hline Ukraine & 56 & 47 & 29 \\
\hline Vereinigte Staaten von Amerika & 65 & 49 & 16 \\
\hline
\end{tabular}

CBOS, BS/12/2010: Stosunek Polaków do innych narodów [Das Verhältnis der Polen zu anderen Nationen]. Warszawa, 01/2010. www. cbos.pl

Veränderungen der Antipathiewerte gegenüber ausgewählten Nationen (\%)

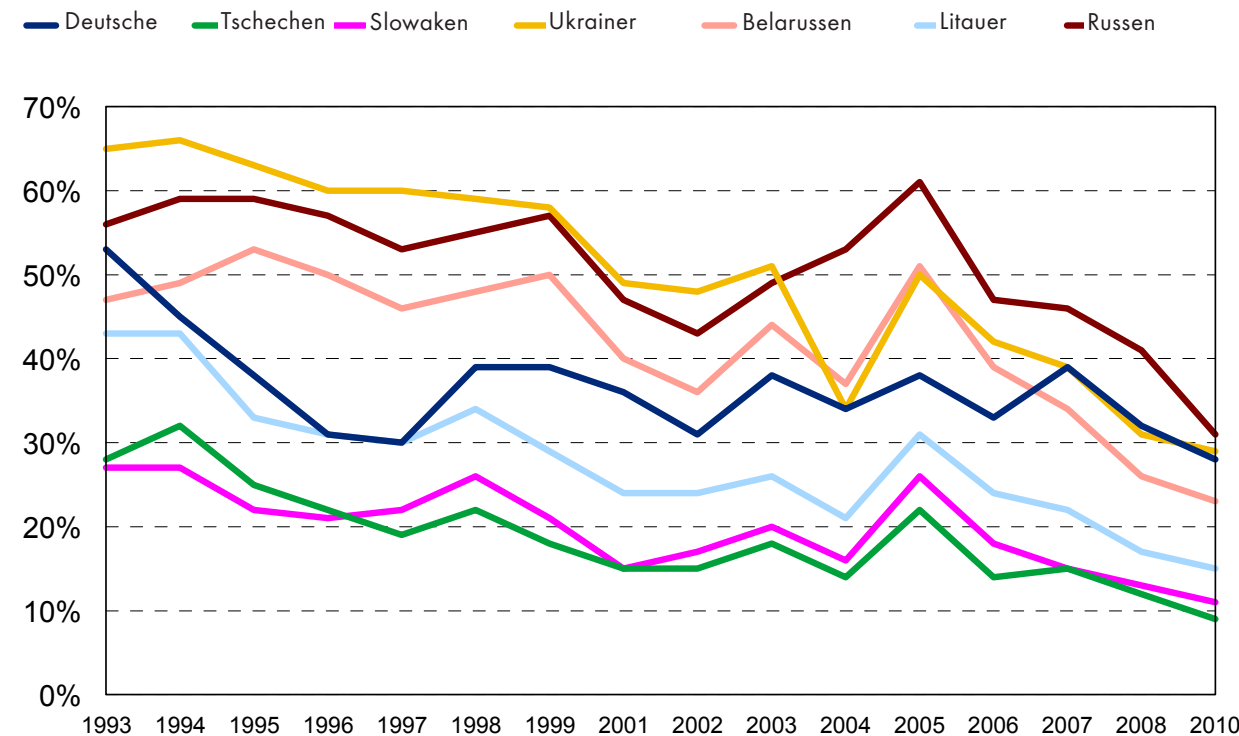

CBOS, BS/12/2010: Stosunek Polaków do innych narodów [Das Verhältnis der Polen zu anderen Nationen]. Warszawa, 01/2010. www.cbos.pl 


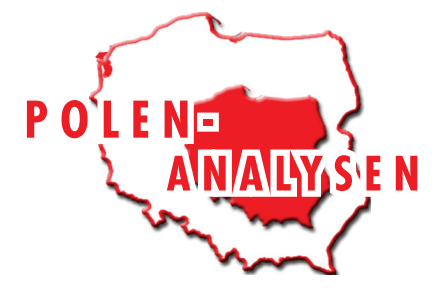

\section{Außenminister Radosław Sikorski im Sejm über die Grundzüge der polnischen Außenpolitik 2010}

\section{Exposé vom 8. April 2010}

Information des Außenministers über die Grundzüge der polnischen Außenpolitik im Jahr 2010

$[\ldots]$

Die europäische Nachbarschaftspolitik hat sich zum Ziel gesetzt, eine freundschaftliche internationale Umgebung aufzubauen. Die Außengrenzen der Europäischen Union sollen keinen Zivilisationssprung markieren. In unserem Verständnis sollte die Europäische Union gegenüber zwei großen Regionen in ihrer Nachbarschaft offen bleiben, der Mittelmeerregion und der östlichen Region. Diese Ziele werden gleichwohl von einer unterschiedlichen Lage bestimmt: Während die südlichen Ränder des Mittelmeers Nachbarn Europas sind, treffen wir im Osten auf europäische Nachbarn.

Gerade diese Wahrnehmung der Nachbarschaft lag der polnisch-schwedischen Initiative der Östlichen Partnerschaft zugrunde. Die Initiative beruht auf der Unterstützung der in den Partnerländern beginnenden Transformationsprozesse, so dass sich ihre Standards dem vom acquis communautaire gesetzten Höchstmaß annähern. Natürlich muss die Basisarbeit der Modernisierung von diesen Ländern selbst geleistet werden.

$[\ldots]$

Wenn wir von den Beziehungen zu Russland sprechen, beobachten wir, dass sie trotz Verbesserungen in den letzten Monaten immer noch von tragischen historischen Inhalten belastet werden. Ich denke, dass unser, d.h. der polnische und russische, Umgang mit dem 70. Jahrestag des Verbrechens von Katyn ein Test dafür ist, inwieweit sich unsere Beziehungen normalisiert haben. Gestern waren wir Zeugen eines historischen Augenblicks, als die Ministerpräsidenten von Polen und Russland gemeinsam der Opfer des Stalinismus gedachten: der polnischen Offiziere, der vom NKWD im Jahr 1940 ermordeten Kriegsgefangenen, aber auch der Russen, Ukrainer, Juden und vieler anderer Nationen der ehemaligen UdSSR, die Säuberungen und anderen Formen sowjetischer Repressionen zum Opfer fielen. Wir sind dafür, das Problem Katyn sowie andere weiße und schwarze Flecken in der Geschichte der polnisch-russischen Beziehungen zu entpolitisieren. Ich danke der Gruppe für schwierige Fragen unter der Leitung von Prof. Adam Daniel Rotfeld und dem Rektor des Moskauer Staatsinstituts für Internationale Beziehungen, Anatolij Torkunov. Diese Gruppe hervorragender Historiker und Experten trug dazu bei, die historische Diskussion auf die Gleise des Dialogs und der Wahrheitssuche zu lenken. Auf diese Weise verlief unser langjähriger Weg der Versöhnung mit den Deutschen, so muss er auch mit den Russen verlaufen. Wenn ich mir die einschneidenden kritischen Äußerungen zum Stalinismus in Erinnerung rufe, die kürzlich von Präsident Medwedew und Ministerpräsident Putin getan wurden, dann glaube ich, dass weitere Gesten und Taten möglich sind. Wir wollen eine bessere wirtschaftliche, kulturelle und wissenschaftliche Zusammenarbeit mit Russland. Das EU-Russland-Projekt der »Modernisierungspartnerschaft«, dessen Grundstein auf dem EU-Russland-Gipfel Ende letzten Jahres in Stockholm gelegt wurde, soll diese Zusammenarbeit befördern.

\section{Hohes Haus!}

Die Ukraine hat gerade eine wichtige Prüfung in Sachen Demokratie bestanden. Die letzten Präsidentschaftswahlen in diesem Land haben gezeigt, wie schnell die dortige Bürgergesellschaft gereift ist. Wir hoffen, dass die ukrainische politische Klasse die Ukraine auf den Weg einer beschleunigten Modernisierung des Staates und der Wirtschaft bringt und die proeuropäische Wahl bestätigt wird, indem die Implementierung der Projekte der Östlichen Partnerschaft vollzogen wird. Wir hoffen, dass die Ukraine, die das institutionalisierte Europa anstrebt, gute Beziehungen und die Zusammenarbeit mit den benachbarten Staaten pflegt. Wie bisher wird Polen Anwalt ukrainischer Angelegenheiten bleiben, wenn die Ukraine dies wünscht.

Mit Aufmerksamkeit und Wohlwollen beobachten wir die Erstarkung der belarussischen Staatlichkeit. Jedoch kann sich in der postsowjetischen Realität Unabhängigkeit nicht ohne Fortschritte der Demokratie verfestigen. Deshalb wollen wir die belarussische Staatsführung weiterhin zu einer Systemveränderung ermutigen und bekräftigen den Willen zu weitgehender Zusammenarbeit. Diese Veränderung sollte in Übereinstimmung mit den Grundsätzen der Demokratie und der Menschenrechte das Recht auf Versammlungsfreiheit beinhalten, was insbesondere die polnische Minderheit in Belarus betrifft - dies fordern wir. 


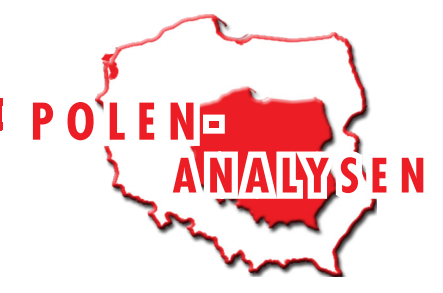

polen-analysen $72 / 10$

Das demokratische Moldawien ist wichtig für Polen und für die Europäische Union. Wir unterstützen die in Chisinau regierende demokratische Koalition, was wir mit Angeboten an Kredithilfen und technischer Hilfe unter Beweis gestellt haben. Darüber hinaus mobilisieren wir unsere Partner in der Europäischen Union für Moldawien.

Unverändert fordern wir die territoriale Integrität Georgiens. Wir ermutigen dazu, den Modernisierungsreformen einen neuen Impetus zu geben. Unsere Erfahrungen haben uns gelehrt, dass dies der sicherste Weg zur Mitgliedschaft in den Institutionen der westlichen Welt ist.

Bei ihren Transformationsbemühungen stellt für Georgien und die übrigen Länder des Südkaukasus Armenien und Aserbaidschan der Anschluss an die Östliche Partnerschaft eine große Chance dar. Wir sehen darüber hinaus die Schlüsselrolle dieser Länder sowie auch der Länder Zentralasiens bei der Schaffung alternativer Lieferwege für Energierohstoffe.

$[\ldots]$

Übersetzung aus dem Polnischen : Silke Plate

Quelle: Ministerstwo Spraw Zagranicznych [Außenministerium], http://www.msz.gov.pl/Expose,2010,34874.html (abgerufen am 14.06. 2010).

Chronik

\section{Vom 1. bis zum 14. Juni 2010}

\begin{tabular}{|c|c|}
\hline 01.06 .2010 & $\begin{array}{l}\text { Das am Vortag dem polnischen Innenminister Jerzy Miller von der bilateralen Untersuchungskommission MAK } \\
\text { in Moskau übergebene Protokoll des Flugschreibers über die letzten Minuten vor dem Flugzeugunglück von } \\
\text { Smolensk am 10. April wird veröffentlicht. Aus den identifizierten Gesprächsbruchstücken geht hervor, dass } \\
\text { sich die Piloten der schlechten Landebedingungen bewusst waren und es sich bei dem Absturz nicht um einen } \\
\text { terroristischen Anschlag gehandelt hat. }\end{array}$ \\
\hline 02.06 .2010 & $\begin{array}{l}\text { Die Sprecherin des Innenministeriums, Małgorzata Woźniak, teilt mit, dass bereits knapp } 193 \text { Mio. Zloty als Hilfe } \\
\text { für Hochwassergeschädigte bereitgestellt worden seien. Für den Wiederaufbau der beschädigten Infrastruktur } \\
\text { werden } 600 \text { Mio. Zloty freigegeben. }\end{array}$ \\
\hline 03.06 .2010 & $\begin{array}{l}\text { Auf einer gemeinsamen Pressekonferenz in Berlin fordern Außenminister Radosław Sikorski und sein deutscher } \\
\text { Amtskollege Guido Westerwelle Israel auf, die Militäraktion Israels von Anfang der Woche aufzuklären, bei } \\
\text { der ein Schiff mit humanitärer Hilfe für den Gaza-Streifen gewaltsam aufgebracht wurde und neun Menschen } \\
\text { ums Leben kamen. Sikorski erklärt, Polen unterstütze das Existenzrecht Israels, aber nicht die Siedlungspolitik } \\
\text { in besetzten Gebieten oder den Einsatz von Gewalt. }\end{array}$ \\
\hline 05.06 .2010 & $\begin{array}{l}\text { Der Parteivorsitzende und Präsidentschaftskandidat von Recht und Gerechtigkeit (Prawo i Sprawiedliwość - } \\
\text { PiS), Jarosław Kaczyński, fordert die Regierung auf, das Haushaltsgesetz angesichts der Hochwasserkatastrophe } \\
\text { in Polen zu novellieren. Die stellvertretende Vorsitzende der Finanzkommission des Sejm, Beata Szydło (PiS), } \\
\text { unterstreicht, dass PiS gegen eine Erhöhung des Haushaltsdefizits sei. Da jedoch von mehr Haushaltseinnahmen } \\
\text { als angenommen auszugehen sei, könnten diese Mittel zur Behebung der Hochwasserschäden eingesetzt werden. }\end{array}$ \\
\hline 06.06 .2010 & $\begin{array}{l}\text { Regierungssprecher Paweł Graś versichert, dass die von Recht und Gerechtigkeit (Prawo i Sprawiedliwość - } \\
\text { PiS) befürwortete Novellierung des Haushaltsgesetzes angesichts der Hochwasserkatastrophe in Polen nicht } \\
\text { notwendig sei. Für Investitionen infolge des Hochwassers stünden 4,127 Mrd. Zloty aus dem Staatshaushalt } \\
\text { zur Verfügung sowie } 560 \text { Mio. Euro aus dem Operativen Programm der Europäischen Union »Infrastruktur } \\
\text { und Umwelt 2007-2013«. }\end{array}$ \\
\hline 06.06 .2010 & $\begin{array}{l}\text { Während einer Messe unter freiem Himmel mit mehreren zehntausend Teilnehmern in Warschau wird Priester } \\
\text { Jerzy Popiełuszko selig gesprochen. Er hatte die Solidarność-Bewegung unterstützt, sich nach der Verhängung } \\
\text { des Kriegsrechts für die Einhaltung der Menschen- und Bürgerrechte eingesetzt, Verfolgten geholfen und in } \\
\text { seiner Warschauer Pfarrgemeinde die bekannten »Messen für das Vaterland« gelesen. Im Oktober } 1984 \text { war er } \\
\text { vom polnischen Sicherheitsdienst gefoltert und ermordet worden. }\end{array}$ \\
\hline
\end{tabular}




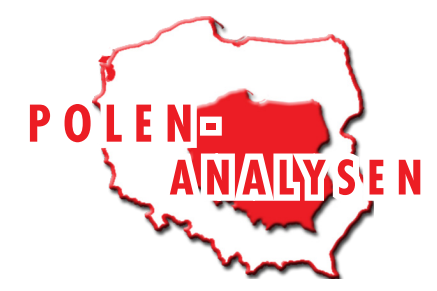

polen-analysen $72 / 10$

\begin{tabular}{|c|c|}
\hline 07.06 .2010 & $\begin{array}{l}\text { Nach einem Treffen mit seinen schwedischen und britischen Amtskollegen, Brigitta Ohlson und David Lidington, } \\
\text { in Stockholm hebt der Staatssekretär für europäische Angelegenheiten, Mikołaj Dowgielewicz, hervor, dass } \\
\text { sich die drei Länder dafür aussprechen, die Diskussion über die Strategie der Europäischen Union in der Wirt- } \\
\text { schafts- und Finanzkrise mit allen } 27 \text { Mitgliedsstaaten zu führen, da die gesamte EU betroffen sei. Der Aus- } \\
\text { schluss der Länder, die nicht der Eurozone angehören, von den Konsultationen sei politisch und wirtschaftlich } \\
\text { unbegründet und gefährlich. }\end{array}$ \\
\hline 08.06 .2010 & $\begin{array}{l}\text { Regierungssprecher Paweł Graś bekräftigt in einem Interview mit dem Radiosender Radio TOK FM, dass } \\
\text { die Regierung derzeit nicht die Notwendigkeit sehe, wegen der Überschwemmungen in Südostpolen und am } \\
\text { Weichsellauf den Katastrophenzustand auszurufen. Darüber hinaus lägen bisher noch keine Hinweise der } \\
\text { Staatlichen Wahlkommission (Państwowa Komisja Wyborcza - PKW) vor, die gegen eine Durchführung der } \\
\text { vorgezogenen Staatspräsidentenwahlen am 20. Juni sprächen. Die Regierung habe ihrerseits die PKW über ihre } \\
\text { Bereitschaft informiert, zusätzliche organisatorische Maßnahmen für die Durchführung der Wahl zu treffen. } \\
\text { Am Vortag hatte der Präsidentschaftskandidat und Parteivorsitzende von Recht und Gerechtigkeit (Prawo i } \\
\text { Sprawiedliwość - PiS), Jarosław Kaczyński, den Ministerpräsidenten aufgefordert, angesichts der drohenden } \\
\text { dritten Überschwemmungswelle den Notstand auszurufen. }\end{array}$ \\
\hline 09.06 .2010 & $\begin{array}{l}\text { Ministerpräsident Donald Tusk stellt gemeinsam mit einer großen Regierungsdelegation in Brüssel die Prioritäten } \\
\text { der polnischen EU-Ratspräsidentschaft in der zweiten Hälfte } 2011 \text { vor. Mit Hinblick auf die Diskussion, wie } \\
\text { der Euro und die EU-Wirtschaft zu retten seien, hebt Tusk hervor, dass sich Polen gegen eine Teilung der } \\
\text { Europäischen Union in besser und schlechter integrierte Mitglieder wende. Es müsse der Grundsatz gewahrt } \\
\text { werden, dass die EU allen Mitgliedsstaaten gleiche Chancen einräumt. }\end{array}$ \\
\hline 09.06 .2010 & $\begin{array}{l}\text { Die Sejmkommission für öffentliche Finanzen spricht sich für die Kandidatur des ehemaligen Ministerpräsidenten } \\
\text { Marek Belka für das Amt des Vorsitzenden der Polnischen Nationalbank (Narodowy Bank Polski - NBP) aus. } \\
\text { Belka war Ende Mai vom amtierenden Staatspräsidenten Bronisław Komorowski nominiert worden, was beim } \\
\text { Koalitionspartner Polnische Bauernpartei (Polskie Stronnictwo Ludowe- PSL) und den Oppositionsparteien } \\
\text { Kritik an Komorowski wegen Kompetenzüberschreitungen ausgelöst hatte. }\end{array}$ \\
\hline 10.06 .2010 & $\begin{array}{l}\text { Der Sejm wählt den ehemaligen Ministerpräsidenten Marek Belka zum Vorsitzenden der Polnischen National- } \\
\text { bank (Narodowy Bank Polski - NBP). }\end{array}$ \\
\hline 10.06 .2010 & $\begin{array}{l}\text { Der Sejm wählt die von der Bürgerplattform (Platforma Obywatelska-PO) nominierte Verwaltungsrechtlerin } \\
\text { Irena Lipowicz, ehemalige Sejmabgeordnete (Freiheitsunion/Unia Wolności-UW) und Botschafterin in Wien, } \\
\text { derzeit Geschäftsführerin der Stiftung für deutsch-polnische Zusammenarbeit, in das Amt der Bürgerrechts- } \\
\text { beauftragten. Lipowicz muss noch vom Senat bestätigt werden. Die Amtszeit dauert fünf Jahre. }\end{array}$ \\
\hline 12.06 .2010 & $\begin{array}{l}\text { Nach dem Tod eines polnischen Soldaten beim Einsatz in Afghanistan kündigt Ministerpräsident Donald Tusk } \\
\text { an, den Abzug der polnischen Truppen aus Afghanistan beim Nato-Gipfeltreffen im November in Lissabon } \\
\text { mit Nachdruck zu thematisieren. }\end{array}$ \\
\hline 13.06 .2010 & $\begin{array}{l}\text { Die Kandidaten der größten Parteien für das Amt des Staatspräsidenten, Bronisław Komorowski (Bürgerplatt- } \\
\text { form/Platforma Obywatelska - PO), Jarosław Kaczyński (Recht und Gerechtigkeit/Prawo i Sprawiedliwość - } \\
\text { PiS), Waldemar Pawlak (Polnische Bauernpartei/Polskie Stronnictwo Ludowe - PSL) und Grzegorz Napieralski } \\
\text { (Demokratische Linksallianz/Sojusz Lewicy Demokratycznej - SLD), nehmen an einer gemeinsamen Fernseh- } \\
\text { debatte teil. }\end{array}$ \\
\hline 13.06 .2010 & $\begin{array}{l}\text { In einem Radiointerview stellt Ministerpräsident Donald Tusk in Aussicht, dass der mit Russland im Januar } \\
\text { ausgehandelte Gasliefervertrag in seinem Umfang noch verändert werden könne, sollten sich die Schiefergasvor- } \\
\text { kommen in Polen als entsprechend groß erweisen. Nach Angaben der Tageszeitung "Rzeczpospolita« sind erste } \\
\text { Schätzungen im Juni zu erwarten, verbindliche Ergebnisse jedoch erst in zwei bis drei Jahren. Der Gaslieferver- } \\
\text { trag mit Russland sieht eine Erhöhung des Gasimports auf } 10,3 \mathrm{Mrd} . \mathrm{m}^{3} \text { und eine Verlängerung bis } 2037 \text { vor. }\end{array}$ \\
\hline 14.06 .2010 & $\begin{array}{l}\text { Der Präsidentschaftskandidat der Bürgerplattform (Platforma Obywatelska-PO) und amtierende Staatspräsident } \\
\text { Bronisław Komorowski unterstreicht, dass die Festlegung eines Datums für die Einführung des Euro in Polen } \\
\text { nicht hilfreich sei. Besser sei ein Orientierungszeitraum, um den Staat für die Übernahme der gemeinsamen } \\
\text { Währung zu mobilisieren. Die Regierung habe derzeit die Jahre } 2012 \text { bis } 2014 \text { angegeben. }\end{array}$ \\
\hline
\end{tabular}




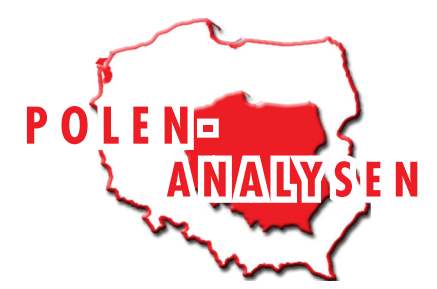

polen-analysen $72 / 10$

Über die Polen-Analysen

Die Polen-Analysen erscheinen zweimal monatlich als E-Mail-Dienst. Sie werden gemeinsam vom Deutschen PolenInstitut Darmstadt, der Bremer Forschungsstelle Osteuropa und der Deutschen Gesellschaft für Osteuropakunde herausgegeben.

Ein Archiv der Polen-Analysen finden Sie im Internet unter www.laender-analysen.de/polen

Kostenloses Abonnement unter http://www.deutsches-polen-institut.de/Newsletter/subscribe.php

\section{Deutsches Polen-Institut Darmstadt}

Das Deutsche Polen-Institut Darmstadt (DPI) ist ein Forschungs-, Informations-, und Veranstaltungszentrum für polnische Kultur, Geschichte, Politik, Gesellschaft und die deutsch-polnischen Beziehungen, die sich im Kontext der europäischen Integration entwickeln. Das seit März 1980 aktive und bis 1997 von Gründungsdirektor Karl Dedecius geleitete Institut ist eine Gemeinschaftsgründung der Stadt Darmstadt, der Länder Hessen und Rheinland-Pfalz sowie des Bundes. Seit 1987 ist die Trägerschaft auf die Kultusminister der Länder ausgedehnt. Einen wesentlichen Beitrag zur Verwirklichung der Institutsziele leisten private Stiftungen. Das DPI hat satzungsgemäß die Aufgabe, durch seine Arbeit zur Vertiefung der gegenseitigen Kenntnisse des kulturellen, geistigen und gesellschaftlichen Lebens von Polen und Deutschen beizutragen.

Ziel der Vermittlertätigkeit des DPI ist es, „die zu interessieren, auf die es politisch, wirtschaftlich, gesellschaftlich und kulturell im deutsch-polnischen Verhältnis ankommt" (Leitlinien 1997). Es geht um die Entscheider und Multiplikatoren in Politik, Kultur, Bildung, Verwaltung, Medien und Wirtschaft und, wesentlich stärker ausgeprägt als bisher, um das Hineinwirken in Wissenschaft, Forschung und Bildung.

Derzeit bemüht sich das DPI in Kooperation mit den verstreuten Orten wissenschaftlicher Polen-Kompetenz an deutschen Hochschulen und Forschungsinstituten verstärkt darum, ausgehend von einer Bestandsaufnahme deutscher Polen-Forschung Ort wissenschaftlicher Forschung und verbindendes, vernetzendes und kooperierendes Zentrum zu werden. Ausgangspunkt der Neuausrichtung ist die kaum mehr kontrollierbare Dynamik des Rückbaus der Ressourcen der wissenschaftlichen Polen-Kompetenz in den unterschiedlichen Disziplinen. Mit der über 55.000 Bände zählenden multidisziplinären Fachbibliothek für Polen mit einer einzigartigen Sammlung polnischer Literatur in der Originalsprache und in deutscher Übersetzung ist das DPI bereits ein geschätzter Ort der Recherche und des wissenschaftlichen Arbeitens. (www.deutsches-polen-institut.de)

\section{Forschungsstelle Osteuropa an der Universität Bremen}

1982 gegründet, widmet sich die Forschungsstelle Osteuropa an der Universität Bremen kulturellen und gesellschaftlichen Entwicklungen der Länder Ost- und Ostmitteleuropas in Zeitgeschichte und Gegenwart. Die Forschungsstelle besitzt in ihrem Archiv eine einzigartige Sammlung alternativer Kulturgüter und unabhängiger Texte aus den ehemaligen sozialistischen Ländern. Darunter befindet sich auch eine umfangreiche Sammlung des "Zweiten Umlaufs", die das Schrifttum und Dokumente unabhängiger Initiativen und gesellschaftlicher Gruppen in Polen aus der Zeit von 1976 bis zum Umbruch umfasst. Neben ausführlicher individueller Forschung zu Dissens und Gesellschaft im Sozialismus, leitet die Forschungsstelle seit Januar 2007 ein gemeinsames Projekt mit einem Verbund von internationalen Forschungsinstituten zum Thema "Das andere Osteuropa - die 1960er bis 1980er Jahre, Dissens in Politik und Gesellschaft, Alternativen in der Kultur. Beiträge zu einer vergleichenden Zeitgeschichte«, welches von der VolkswagenStiftung finanziert wird.

Im Bereich der post-sozialistischen Gesellschaften sind in den letzten Jahren umfangreiche Forschungsprojekte durchgeführt worden, deren Schwerpunkte auf politischen Entscheidungsprozessen, Wirtschaftskultur und der EU-Osterweiterung lagen. Eine der Hauptaufgaben der Forschungsstelle ist die Information der interessierten Öffentlichkeit. Dazu gehören unter anderem regelmäßige E-Mail-Informationsdienste mit fast 15.000 Abonnenten in Politik, Wirtschaft und den Medien.

Mit ihrer in Deutschland einzigartigen Sammlung von Publikationen zu Osteuropa ist die Forschungsstelle eine Anlaufstelle sowohl für Wissenschaftler als auch für die interessierte Öffentlichkeit. In der Bibliothek sind derzeit neben anderen breit angelegten Beständen allein aus Polen ca. 300 laufende Periodika zugänglich. Die Bestände werden in Datenbanken systematisch erfasst. (www.forschungsstelle.uni-bremen.de)

Die Meinungen, die in den Polen-Analysen geäußert werden, geben ausschließlich die Auffassung der Autoren wieder. Abdruck und sonstige publizistische Nutzung sind nach Rücksprache mit der Redaktion gestattet.

Redaktion: Prof. Dr. Dieter Bingen (Darmstadt), Silke Plate, M.A. (Bremen) Technische Gestaltung: Matthias Neumann

Polen-Analysen-Layout: Cengiz Kibaroglu, Matthias Neumann

Die Polen-Analysen werden im Rahmen der Datenbank World Affairs Online (WAO) ausgewertet und sind im Portal IREON www.ireon-portal.de recherchierbar.

ISSN 1863-9712 @ 2010 by Deutsches Polen-Institut Darmstadt und Forschungsstelle Osteuropa, Bremen

Kontakt: Dr. Andrzej Kaluza, Presse- und Öffentlichkeitsarbeit, Deutsches Polen-Institut, Mathildenhöhweg 2,

D-64287 Darmstadt, Tel.: 06151/4985-13, Fax: 06151/4985-10, E-Mail: polen-analysen@dpi-da.de, Internet: www.laender-analysen.de/polen 